\title{
repisälud
}

This is the peer reviewed version of the following article:

\section{El estado de la prevención cardiovascular en España}

Miguel Ángel Royo-Bordonada ${ }^{\mathrm{a}}$, José Maria Lobos ${ }^{\mathrm{b}}$, Carlos Brotons ${ }^{\mathrm{b}}$, Fernando Villar ${ }^{\mathrm{c}}$, Carmen de Pablo ${ }^{\mathrm{d}}$, Pedro Armario ${ }^{\mathrm{e}}$, Olga Cortés ${ }^{\mathrm{f}}$, Antonio Gil Nuñez ${ }^{\mathrm{g}}$, Ángel Lizcano $^{\mathrm{h}}$, Ana de Santiago ${ }^{\mathrm{i}}$, Susana Sans, en nombre del Comité Español Interdisciplinario de Prevención Cardiovascular (CEIPC)*

Med Clin (Barc) , 142 (1), 7-14

which has been published in final form at http://dx.doi.org/10.1016/j.medcli.2012.09.046 


\section{repisälud}

\section{El estado de la prevención cardiovascular en España}

Miguel Ángel Royo-Bordonada ${ }^{\mathrm{a}}$, José Maria Lobos ${ }^{\mathrm{b}}$, Carlos Brotons ${ }^{\mathrm{b}}$, Fernando Villar ${ }^{\mathrm{c}}$, Carmen de Pablo $^{\mathrm{d}}$, Pedro Armario ${ }^{\mathrm{e}}$, Olga Cortés ${ }^{\mathrm{f}}$, Antonio Gil Nuñez ${ }^{\mathrm{g}}$, Ángel Lizcano ${ }^{\mathrm{h}}$, Ana de Santiago ${ }^{\mathrm{i}}$, Susana Sans ${ }^{j}$, en nombre del Comité Español Interdisciplinario de Prevención Cardiovascular (CEIPC)*

${ }^{a}$ Instituto de Salud Carlos III. Madrid, España.

${ }^{\mathrm{b}}$ Sociedad Española de Medicina de Familia y Comunitaria. Madrid, España.

${ }^{\mathrm{c}}$ Sociedad Española de Arteriosclerosis. Madrid, España.

${ }^{\mathrm{d}}$ Sociedad Española de Cardiología. Madrid, España.

${ }^{\text {e }}$ Sociedad Española de Hipertensión-Liga Española de la Lucha Contra la HTA. Barcelona, España.

${ }^{\mathrm{f}}$ Asociación Española de Pediatría de Atención Primaria. Madrid, España.

${ }^{g}$ Sociedad Española de Neurología. Barcelona, España.

${ }^{\mathrm{h}}$ Federación de Asociaciones de Enfermería Comunitaria y Atención Primaria. Madrid, España.

${ }^{\mathrm{i}}$ Sociedad Española de Médicos de Atención Primaria. Madrid, España.

${ }^{j}$ Sociedad Española de Salud Pública y Administración Sanitaria. Barcelona, España.

Autor para correspondencia: Miguel Ángel Royo Bordonada. Escuela Nacional de Sanidad. Instituto de Salud Carlos III. c/ Sinesio Delgado 8. 28029 Madrid. Correo-e: mroyo@isciii.es Tel: 918222274

* Miembros del CEIPC: José María Lobos (Sociedad Española de Medicina de Família y Comunitaria), Miguel Angel Royo (Instituto de Salud Carlos III), Carlos Brotons (Sociedad Española de Medicina de Familia y Comunitaria), Rosa Moreno (Sociedad Española de Angiología y Cirugía Vascular), Antonio Pérez Pérez (Sociedad Española de Diabetes), Susana Sans (Sociedad Española de Salud Pública y Administración Sanitaria), Ana de Santiago (Sociedad Española de Médicos de Atención Primaria), Carmen de Pablo (Sociedad Española de Cardiología), Miguel Camafort Babkowski (Sociedad Española de Medicina Interna), Juan Pedro-Botet Montoya (Sociedad Española de Arteriosclerosis), Roberto Elosua (Sociedad Española de Salud Pública y Administración Sanitaria), Alberto Cordero (Sociedad Española de Cardiología), Francisco Fornés Ubeda (Sociedad 


\section{repisåalud}

Española de Medicina y Seguridad en el Trabajo), Antonio Gil Nuñez (Sociedad Española de Neurología), Vicenta Lizarbe (Dirección General de Salud Pública. Ministerio de Sanidad y Consumo), Antonio Maiques (Sociedad Española de Medicina de Família y Comunitaria), Fernando de Álvaro Moreno (Sociedad española de Nefrología), Pedro Armario (Sociedad Española de Hipertensión-Liga Española de la Lucha Contra la HTA), Olga Cortés Rico (Asociación Española de Pediatría de Atención Primaria), Fernando Villar (Sociedad Española de Arteriosclerosis), Ángel Lizcano (Federación de Asociaciones de Enfermería Comunitaria y Atención Primaria). 


\section{repisälud}

\section{RESUMEN}

\section{Introducción}

En España, donde las enfermedades cardiovasculares constituyen la primera causa de muerte, el control de factores de riesgo es bajo. Este estudio analiza la implantación de la valoración del riesgo cardiovascular ( $\mathrm{RCV}$ ) en la práctica clínica y la existencia de objetivos de control entre los indicadores de calidad asistencial y los sistemas de incentivación profesional.

\section{Métodología}

Entre 2010 y 2011, mediante un cuestionario diseñado ad hoc, se recogió información de cada Comunidad Autónoma (CCAA) sobre prevalencia y control de los principales factores de RCV, y valoración del RCV y objetivos de control entre los indicadores de calidad y los sistemas de incentivación de atención primaria. Quince de las 17 CCAA respondieron al cuestionario.

\section{Resultados}

El RCV se calcula con SCORE en 9 CCAA, REGICOR en 3 y Framingham en 3, con coberturas del 3,4 \% al 77,6\%. El control de los factores de RCV fue en general bajo y variable: hipertensión (22,7-61,3\%), dislipemia (11-45,1\%), diabetes (18,5-84\%) y tabaquismo (20-50,5\%). Muchas CCAA no incluyeron el RCV entre los indicadores de calidad asistencial o los sistemas de incentivación, escaseando las iniciativas sobre estilos de vida.

\section{Conclusiones}

Existe variabilidad en las políticas de prevención cardiovascular de las CCAA. Convendría extender la implantación de una guía consensuada de prevención cardiovascular, la valoración del RCV en la historia clínica electrónica (HCE), haciendo especial énfasis en los estilos de vida, y la 


\section{repisälud}

incorporación de la valoración y control del RCV entre los indicadores de calidad asistencial y los sistemas de incentivación profesional.

Palabras clave: prevención cardiovascular, riesgo cardiovascular, calidad asistencial. 


\section{repisälud}

SUMMARY

\section{Introduction}

In Spain, where cardiovascular diseases are the leading cause of death, control of their risk factors is low. This study analyzes the implementation of cardiovascular risk (CVR) assessment in clinical practice and the existence of control objectives amongst quality care indicators and professional incentive systems.

\section{Methodology}

Between 2010 and 2011, data from each autonomous community was collected, by means of a specific questionnaire concerning prevalence and control of major CVR factors, CVR assessment, and implementation of control objectives amongst quality care indicators and primary care incentive systems.

\section{Results}

Fifteen out of 17 autonomous communities filled in the questionnaire. CVR was calculated through SCORE in 9 autonomous communities, REGICOR in 3 and Framingham in 3, covering 3,4 $\%$ to $77,6 \%$ of target population. The resulting control of the main CVR factors was low and variable: hypertension (22,7-61,3\%), dyslipidemia (11-45,1\%), diabetes (18,5-84\%) and smoking (20-50,5\%). Most autonomous communities did not consider CVR assessment and control amongst quality care indicators or incentive systems, highlighting the lack of initiatives on lifestyles.

\section{Conclusions}

Variability exists in cardiovascular prevention policies among autonomous communities. It is necessary to implement a common agreed cardiovascular prevention guide, to encourage physicians 


\section{repisälud}

to implement CVR on electronic clinical history, and to promote CVR assessment and control inclusion amongst quality care indicators and professional incentive systems, focusing on lifestyles management.

Key words: cardiovascular prevention, cardiovascular risk, quality care. 


\section{repisälud}

\section{Introducción}

En España, las enfermedades cardiovasculares (ECV) constituyen la primera causa de muerte, con un tercio del total de defunciones, principalmente por cardiopatía isquémica (31\%) y enfermedad cerebrovascular $(28 \%)^{1}$. Además del impacto sobre la salud y la calidad de vida de los ciudadanos, los costes de las ECV se estiman en torno a 2.000 millones de euros anuales ${ }^{2}$.

Las tasas de mortalidad ajustadas por edad para la ECV llevan disminuyendo en España desde 1975 al 3,1\% anual $^{1}$. Un 50\% de este descenso podría deberse a la reducción de algunos factores de riesgo, como el colesterol total y la presión arterial sistólica ${ }^{3}$, pese a que su control sigue siendo muy bajo en España ${ }^{4-6}$. Sin embargo, las tendencias negativas de otros factores de riesgo, como obesidad ${ }^{7}$ y diabetes mellitus ${ }^{8}$, la inactividad física en varones y el tabaquismo en mujeres ${ }^{3}$, junto con la mayor supervivencia de los pacientes y el envejecimiento de la población, hacen que, en cifras absolutas, el impacto de las ECV siga aumentando ${ }^{1}$.

Las diferencias territoriales en la prevalencia y grado de control de factores de riesgo cardiovascular en España ${ }^{9}$, coherentes con el patrón geográfico de mortalidad cardiovascular ${ }^{1}$, indican la existencia de un amplio margen para la prevención. La variabilidad en el cumplimiento de objetivos terapéuticos podría relacionarse en parte con diferencias regionales en el grado de implantación y adherencia a las guías de prevención cardiovascular. Además, las estrategias y prioridades de tratamiento en España no siempre están determinadas por el riesgo cardiovascular global $(\mathrm{RCV})^{10}$, pese a que tal abordaje resulta en un mejor control de factores de riesgo ${ }^{11,12}$. Por ello, diversos grupos de expertos abogan por un enfoque integral de la ECV, incorporando la valoración del RCV y su control entre los indicadores de calidad asistencial ${ }^{13-15}$.

El Comité Español Interdisciplinario para la Prevención Cardiovascular (CEIPC) adapta regularmente las guías europeas de prevención cardiovascular a la realidad española, transmitiendo a 


\section{repisälud}

los profesionales sanitarios un enfoque común para mejorar la calidad de la práctica clínica ${ }^{15}$, centrando sus mayores esfuerzos en acciones encaminadas a promover la implementación de las guías y reducir la variabilidad en el abordaje de las personas con RCV. Con este estudio, el CEIPC pretende analizar posibles diferencias regionales en la implantación de la valoración del RCV en la práctica clínica, la existencia de objetivos de control del mismo y su incorporación entre los indicadores de calidad asistencial y los sistemas de incentivación de los profesionales de Atención Primaria. 


\section{repisälud}

\section{Metodología}

En mayo de 2009, establecidos los objetivos del proyecto, el CEIPC designó a un grupo de expertos para diseñar un cuestionario ad hoc. El grupo, mediante un método de brainstorming estructurado, basado en la técnica de Metaplan ${ }^{16}$, debatió la estructura y los contenidos generales del cuestionario. Este método persigue la reflexión individual y la participación libre y ordenada de todos los componentes del grupo, para presentar el conocimiento, fundamentado en las mejores evidencias disponibles, estructurando las aportaciones de los presentes e identificando consensos y disensos. A continuación, en una fase de trabajo individual, tras distribuir los diferentes apartados del cuestionario por áreas temáticas entre los participantes, cada componente del grupo elaboró una propuesta preliminar de ítems, que se distribuyó al resto por correo electrónico. Finalizado el trabajo individual, el grupo de expertos se reunió de nuevo debatir y aprobar los contenidos definitivos de la encuesta, siguiendo el método previamente descrito. Finalmente, el cuestionario fue presentado al resto de miembros del CEIPC para su validación definitiva.

El cuestionario fue diseñado para aplicarse en las Comunidades Autónomas (CCAA). Las ciudades autónomas de Ceuta y Melilla fueron excluidas por no haber recibido las transferencias de asistencia sanitaria. El cuestionario, que incluía una breve descripción de los objetivos del estudio, los resultados esperados y la recomendación de proporcionar las fuentes de información, las vías de acceso a las mismas y cualquier información adicional considerada relevante, constaba de 44 preguntas agrupadas en 3 apartados: El primero sobre desarrollo de programas de prevención cardiovascular y actuaciones específicas para difundir sus recomendaciones y facilitar su aplicación; el segundo sobre el RCV y la presencia de objetivos e indicadores de valoración y control del mismo entre los indicadores de calidad asistencial y los sistemas de incentivación de atención primaria; y el tercero sobre los principales factores de riesgo cardiovascular (hipertensión, dislipemia, diabetes, 


\section{repisälud}

tabaquismo, obesidad e inactividad física) y la existencia de estimadores de prevalencia y control de los mismos.

El cuestionario fue enviado por el CEIPC a los Directores de Asistencia Sanitaria y/o Atención Primaria de las Consejerías de Salud de las CCAA en Enero de 2010, realizando hasta tres recordatorios por electrónico y/o teléfono. A 31 de Julio de 2010 se habían recibido 9 de los 17 cuestionarios $(52,9 \%)$. En los meses siguientes el CEIPC estableció contactos adicionales con responsables de asistencia sanitaria y/o profesionales de referencia en prevención cardiovascular de las CCAA no respondedoras. Con aquellas Comunidades que así lo requirieron, los coordinadores del CEIPC mantuvieron una entrevista personal para presentar el proyecto. El 30 de junio de 2011, 15 CCAA habían proporcionado toda o parte de la información solicitada (tasa de respuesta 88,2\%), dándose por finalizada la fase de recogida de la información. El cuestionario fue cumplimentado por profesionales de la salud con diversos niveles de responsabilidad en las unidades de planificación asistencial, gestión clínica y calidad, y los servicios de Atención Primaria y Especializada de los Servicios Regionales de Salud. Tras el análisis de los datos, se procedió a la redacción de un informe preliminar que fue remitido a las CCAA para su conocimiento, revisión y, en su caso, corrección de errores. 


\section{repisälud}

\section{RESULTADOS}

\section{Programas de prevención cardiovascular}

Catorce de las quince CCAA que respondieron disponen de un programa que orienta sus actuaciones en prevención cardiovascular; mientras que Cantabria sigue las recomendaciones recogidas en las estrategias de salud del Ministerio de Sanidad. Aunque todas las CCAA disponen de guías de práctica para orientar las actuaciones clínicas en prevención cardiovascular, Cantabria, Madrid, Murcia y Galicia remiten a la Cartera de Servicios de Atención Primaria o a sus Normas Técnicas Mínimas, mientras que Aragón remite a las Guías Clínicas del SNS.

\section{Valoración y abordaje del RCV (Tabla 1)}

El método recomendado para calcular el RCV fue SCORE -solo o combinado- en 9 CCAA (60\%), REGICOR en $3(20 \%)$ y Framingham en las 3 restantes (20\%). Todas las CCAA, excepto Cantabria, tenían definidas población objetivo y periodicidad con la que debía valorarse el RCV, y en todas, excepto Extremadura y Galicia, está implementado el cálculo del RCV en la Historia Clínica Electrónica (HCE). Sin embargo, el porcentaje de población diana a la que se realiza la valoración del RCV, en las 8 CCAA que aportaron este dato, varió del 3,4 \% de Asturias al 77,6\% de Cataluña. La determinación de modificadores del RCV y/o lesión de órganos diana se encontraba implementada en la HCE de 8 CCAA.

De las 8 CCAA que contemplan la valoración del RCV entre los indicadores de calidad asistencial, 6 aportaron el indicador, que fue el porcentaje de valoración del RCV en la HCE, pero estimado en diferentes grupos de pacientes: en Canarias, pacientes con algún factor mayor de RCV, en Cataluña, pacientes con hipercolesterolemia, en Galicia, pacientes con hipertensión arterial (HTA), y sin especificar en Aragón, Asturias y País Vasco. En las 5 CCAA que proporcionaron el estándar esperado, este varió del 10\% de Asturias al 100\% de Canarias. 


\section{repisälud}

De las 7 CCAA que incluyen la cobertura de la valoración del RCV en el sistema de incentivos de Atención Primaria, solo Asturias contempla objetivos de tratamiento en pacientes de alto riesgo y el País Vasco de reducción del RCV.

\section{Valoración y abordaje de la HTA (Tabla 2)}

La prevalencia de HTA en población adulta, en las once CCAA que aportaron una estimación, osciló del 15\% de Andalucía al 35\% de Canarias. En Baleares fue del 47,8\% en población de 35-74 años. El porcentaje del total de hipertensos (tratados o no) en los que se alcanzaron objetivos de control, en las 5 CCAA que proporcionan este dato, osciló del 22,7\% de Valencia al 61,3\% de Cataluña, aunque Cataluña consideró buen control cifras por debajo de 160/95 en >14 años.

De las 7 CCAA que contemplaron un indicador de control de la HTA (porcentaje de pacientes controlados), solo Cataluña y Murcia incorporaron un segundo indicador, para pacientes con RCV elevado y diabetes o insuficiencia renal respectivamente. El estándar esperado varió del 33\% de Madrid al 64,6\% de Cataluña (52,9\% en pacientes con RCV elevado). En Murcia el objetivo fue conseguir una mejora del 10\% en el control respecto al año anterior. Asturias, Baleares y País Vasco incluyen en el sistema de incentivos de Atención Primaria el porcentaje de población con RCV alto en el que se alcanzan objetivos de control de tensión arterial.

\section{Valoración y abordaje de la dislipemia (Tabla 3)}

La prevalencia de dislipemias osciló del 12\% de Murcia al 31,3\% de Canarias en las diez CCAA que proporcionan el dato, aunque los criterios diagnósticos y los grupos de edad fueron heterogéneos. Los porcentajes del total de dislipémicos (tratados o no) en los que se alcanzaron objetivos de control fueron del 11\% en Murcia, 19,2\% en Valencia y 45,1\% en el País Vasco.

Tres CCAA (Islas Baleares, Murcia y País Vasco) contemplaron, entre sus indicadores de calidad asistencial, el porcentaje de pacientes con buen control del colesterol; mientras Cataluña y 


\section{repisälud}

Madrid incluyeron el porcentaje de pacientes coronarios con colesterol LDL (c-LDL) por debajo de 120 y $100 \mathrm{mg} / \mathrm{dl}$ respectivamente. Cataluña incluyó además el porcentaje de pacientes con enfermedad cerebrovascular y c-LDL $<120$. El estándar esperado varió del 13\% de Murcia al $64 \%$ de Madrid. Baleares y País Vasco contemplan en el sistema de incentivos de Atención Primaria el porcentaje de población con RCV alto en la que se alcanzan objetivos de control del colesterol total o c-LDL.

\section{Valoración y abordaje de la diabetes (Tabla 4)}

En las 10 CCAA que aportaron datos de prevalencia de diabetes, ésta osciló del 4,8\% de Baleares al 10,6\% de Canarias. De éstas, 7 proporcionaron el porcentaje del total de diabéticos (tratados o no) en los que se alcanzaron objetivos de control, desde el 18,5\% de Valencia al $84 \%$ de Baleares. De las 10 CCAA que contemplaron, entre sus indicadores de calidad asistencial, el porcentaje de diabéticos controlados (Canarias solo en algunas Áreas de Salud), 6 utilizaron valores de HbAlc menor o igual de 7 como indicador de buen control; mientras en Galicia el límite aceptable fue menos de 7,5, en Canarias y Cataluña menos de 8 y el País Vasco no especificó ningún valor. Además, Andalucía contempla el porcentaje de pacientes diabéticos con HbAlc mayor de 8, Asturias el porcentaje con determinación anual del índice albúmina/creatinina y Cataluña el porcentaje con cribado de pie diabético y retinopatía. El estándar esperado para el control de las cifras de HbAlc, en las 5 CCAA que informaron del mismo, osciló del 23,6\% de Valencia al 40-70\% de Aragón.

\section{Valoración y abordaje del tabaquismo (Tabla 5)}

En las 9 CCAA que proporcionan datos de prevalencia de tabaquismo (4 con datos de la Encuesta Nacional de Salud -ENS-), ésta osciló del 24,8\% de Galicia al 33,9\% de Murcia. La prevalencia registrada en la HCE fue del 20,3\% en Cataluña y del 3,1\% en Valencia, frente al 24,8\% estimado por la ENS. 


\section{repisålud}

El porcentaje de fumadores que abandonan el hábito tabáquico, en las 5 CCAA que proporcionaron el dato, osciló del 7,5\% de Cataluña (que considera nuevos abandonos en los últimos 12 meses) al 50,5\% del País Vasco. Canarias incorpora el porcentaje de abandono entre sus indicadores de calidad asistencial y Asturias en el sistema de incentivos de Atención Primaria. En Navarra y en 3 áreas de Murcia financiaban las terapias farmacológicas de deshabituación. En 8 CCAA disponen de una (Galicia y Aragón) o más unidades de tabaquismo.

\section{Valoración y abordaje de la obesidad y ejercicio físico (Tabla 6)}

La prevalencia de obesidad, en las 9 CCAA que proporcionaron el dato, osciló del 12\% de Aragón al 27,8\% de Canarias. En Valencia, la prevalencia registrada en la HCE fue del 3,9\%, frente al 14,5\% estimado por la ENS. Todas las CCAA incorporan datos antropométricos (talla y peso) en su HCE para el cálculo del Índice de Masa Corporal, y todas excepto Baleares, incorporan también el perímetro abdominal. La prevalencia de sedentarismo, en las 3 CCAA que proporcionaron este dato, fue del $31,1 \%$ en Valencia, $45,1 \%$ en Galicia y, en Murcia, del $45 \%$ en hombres y $52 \%$ en mujeres. En 10 CCAA existe un programa de actuaciones concretas para fomentar el ejercicio físico. Ninguna CCAA incluye el control de la obesidad, dieta o ejercicio físico entre sus indicadores de calidad asistencial y el sistema de incentivos en Atención Primaria. 


\section{repisälud}

\section{DISCUSIÓN}

Todas las CCAA disponen de un programa y/o guía de práctica clínica en prevención cardiovascular. La mayoría (60\%) siguen las recomendaciones del CEIPC y calculan el RCV con SCORE, siendo REGICOR y Framingham otras tablas usadas con frecuencia. El control de los principales factores de riesgo cardiovascular fue escaso y variable, y la mayoría de las CCAA no contemplaron de forma sistemática la valoración y el control del RCV entre sus indicadores de calidad asistencial y sistemas de incentivación de profesionales de Atención Primaria.

Pese a la elevada implementación del RCV en la HCE, la cobertura del mismo fue baja -solo Cataluña alcanzó el estándar esperado- y sujeta a considerable variabilidad. Esto puede deberse a la existencia de múltiples HCE, con diferentes formas de calcular el RCV, como revela la presencia variable de modificadores de riesgo, sujetas a grados variables de dificultad en su manejo. De acuerdo con resultados todavía no publicados de un estudio del CEIPC sobre barreras a la implementación de las guías, en todas las CCAA, un porcentaje variable, en torno al 20\%, de médicos de Atención Primaria declararon que el principal motivo por el cual no realizaban el cálculo de RCV, después de la falta de tiempo, era que no disponían del programa informático adecuado. Por término medio, las CCAA con cifras más altas de cobertura de valoración del RCV fueron las que incluyeron este indicador en el sistema de incentivos de Atención Primaria, en consonancia con los resultados de un estudio europeo, que observó una mayor adherencia a las guías entre los médicos de Atención Primaria del Reino Unido, donde su remuneración estaba ligada a ese concepto ${ }^{17}$.

Con la excepción de Baleares y Canarias, la prevalencia estimada de HTA se sitúo entre el 15 y el $25 \%$, claramente inferior a las cifras del estudio ENRICA (estudio poblacional de ámbito nacional realizado en España de 2008 a 2010) ${ }^{4}$ y otros estudios previos a nivel nacional ${ }^{18}$. Por el contrario, el control de la HTA fue superior al 22\% del estudio ENRICA ${ }^{4}$ en todas las comunidades que proporcionaron el dato. El mejor control de Cataluña cabe atribuirlo al diferente estándar 


\section{repisälud}

utilizado. En todos los casos, el control de la HTA fue inferior al estándar esperado, y solo 3 CCAA incorporaron dicho control en el sistema de incentivos de Atención Primaria. Un reciente metaanálisis llevado a cabo en España ${ }^{18}$ ha confirmado que el control de la HTA dista de ser óptimo, no habiendo mejorado en los últimos años. Por ello, se han publicado documentos de consenso en Europa $^{19}$ y España ${ }^{20}$ que analizan el problema y proponen cambios en las políticas de salud para lograr una mejora del control de la HTA.

Las diferencias en las fuentes de información (criterios diagnósticos y grupos de edad, tipo y ámbito de estudio) pueden justificar en parte las variaciones entre CCAA en la prevalencia de dislipemias y el hecho de que las cifras (por debajo del 25\% excepto en Canarias) sean relativamente bajas en comparación con las del estudio ENRICA, que obtuvo una prevalencia de colesterol total > $200 \mathrm{mg} / \mathrm{dl}$ del 50,3\% en población mayor de 18 años $^{4}$. Destaca la diferencia en el control de la hipercolesterolemia (entre el 11 y el 45,1\%) entre las tres CCAA que aportaron este dato. En el ENRICA $^{4}$, el 52,9\% de los tratados estaban controlados, lo que supone el 11,1\% de toda la población con dislipemia. Además, los indicadores de control utilizados son diferentes entre CCAA y tan solo Baleares y el País Vasco los tienen en cuenta en los sistemas de incentivos de Atención Primaria.

La prevalencia estimada de diabetes osciló entre el 5 y el 10\%, por debajo del 13,8\% estimado en el reciente Estudio Di@bet.es ${ }^{8}$. El indicador principal de control de la diabetes fue una HbA1C menor de 7\% en la mayoría de las CCAA, en consonancia con la evidencia científica disponible y las recomendaciones del $\mathrm{CEIPC}^{21}$, detectándose una gran variación interterritorial en el grado de control metabólico. Aunque los indicadores de control de la diabetes están más extendidos que los del resto de factores de riesgo, tan solo las comunidades de Asturias y Cataluña recogieron indicadores de daño orgánico (microalbuminuria y retinopatía, respectivamente), destacando la ausencia de indicadores de hábitos de vida (alimentación, ejercicio) y del control de otros factores de riesgo 


\section{repisälud}

mayores, como HTA, dislipemia y tabaquismo, en pacientes diabéticos, aspectos de gran relevancia clínica y pronóstica.

La prevalencia de tabaquismo en las CCAA se situó en torno al 25\%, con cifras en Canarias, Cataluña y Comunidad Valenciana menores que las estimadas en la ENS $2006^{22}$, aunque en el caso de Cataluña son datos de la HCE. Estas cifras son similares a las del estudio ENRICA ${ }^{4}$, más reciente. La discordancia en las estimaciones de tabaquismo de la Comunidad Valenciana, con datos de prevalencia del $3,1 \%$ en la HCE y del $24,8 \%$ en la ENS -tan marcada como la que se aprecia para la estimación de la obesidad-, conjuntamente con la falta de datos de otras CCAA, ponen en duda la correcta cumplimentación de la HCE. Aunque casi todas las CCAA disponen de guías o protocolos de abordaje del tabaquismo y consideran prioritario el control del mismo, sólo Asturias y Canarias contemplan entre sus indicadores de calidad asistencial y en su sistema de incentivos el porcentaje de abandono en pacientes con RCV elevado. Quizás por este motivo los porcentajes de abandono no son bien conocidos. De las cinco CCAA que ofrecieron datos de deshabituación tabáquica, salvo en Murcia, con un 20\%, las cifras declaradas fueron superiores al $20,5 \%$ de la ENS $2006^{22}$ y al $24,6 \%$ del estudio ENRICA ${ }^{4}$. Además, las terapias farmacológicas de deshabituación se financiaban exclusivamente en Navarra y parcialmente en Murcia, y solo 8 comunidades declaran disponer de unidades de tabaquismo. Considerando la todavía elevada prevalencia de fumadores en nuestro medio y que entre el $10 \%{ }^{23}$ y el $19 \%^{24}$ de los mismos continúan con el hábito tras un episodio cardiovascular, los recursos ofrecidos por la sanidad pública parecen claramente insuficientes. Una iniciativa para paliar esta deficiencia es la Estrategia Euskadi Libre del Humo del Tabaco, lanzada en 2010 en el País Vasco, con la que se pretende crear unidades de deshabituación en los centros de Atención Primaria en los que la prevalencia de tabaquismo sea más elevada ${ }^{25}$.

La prevalencia estimada de obesidad osciló entre el 12\% de Aragón y el 27,8\% de Canarias, siendo en su conjunto sensiblemente inferior a la encontrada en el estudio ENRICA, con datos de 


\section{repisälud}

peso y talla medidos ${ }^{7}$. A esta infravaloración del problema, hay que añadir la baja tasa de registro en la HCE, como sugieren las cifras de Valencia, pese a la generalización de la implementación de los datos antropométricos en la HCE. La mayor parte de las CCAA disponen de algún programa de ejercicio físico, lo que podría contribuir a explicar el reciente descenso observado en el sedentarismo en población adulta de Girona ${ }^{26}$ y en población anciana española ${ }^{27}$. Sin embargo, la existencia de estudios que muestran la tendencia contraria ${ }^{28}$ y la todavía elevada prevalencia de sedentarismo, entre un tercio y más de la mitad de la población adulta, junto con el progresivo y alarmante incremento de las cifras de obesidad ${ }^{29}$, demandan una respuesta contundente de las administraciones en este ámbito. Los médicos, más habituados al tratamiento de la enfermedad que a su prevención, necesitan formación en el manejo de los estilos de vida, que aumente su habilidad y confianza en esta área, y motivación para cambiar el paradigma de la atención médica hacia la prevención. Difícilmente podrá alcanzarse este objetivo mientras la dieta, los niveles de actividad física y la obesidad no se contemplen entre los indicadores de calidad asistencial y los sistemas de incentivos de Atención Primaria. Los resultados del estudio Whitehall II señalan que los cambios en la dieta y los niveles de actividad física reducen de forma independiente las cifras de colesterol, apoyando la importancia de reforzar el manejo de los estilos de vida en la estrategia de prevención cardiovascular ${ }^{30}$.

El nivel de información proporcionado por las 15 CCAA participantes fue muy variable. Esto podría deberse al diferente perfil y grado de responsabilidad de las personas que cumplimentaron el cuestionario, pues algunos servicios regionales de salud no tenían identificado un profesional de referencia en prevención cardiovascular. La escasez de información sobre las fuentes originales de los datos, la diversidad de indicadores utilizados y las diferencias temporales en la recogida de datos, debidas a la reducida tasa de respuesta obtenida en una primera instancia $(52,9 \%)$, limitan en parte las comparaciones realizadas; aunque no impiden la extracción de conclusiones razonablemente válidas y claramente relevantes para el conjunto del Sistema Nacional de Salud. 


\section{repisälud}

Aunque la mayoría de las CCAA aplican las recomendaciones del CEIPC, todavía existe cierta variabilidad en las guías y tablas de cálculo utilizadas, detectándose una escasa y heterogénea estratificación del RCV, junto con una baja tasa de registro de los principales factores de riesgo en la HCE. El control del RCV es variable entre CCAA y tiene escasa presencia entre los indicadores de calidad asistencial y en los sistemas de incentivos de Atención Primaria. Además, se presta poca atención a la modificación de estilos de vida y obesidad, ausentes entre los indicadores de calidad asistencial y en los sistemas de incentivos. En conjunto, aunque estos datos sugieren un compromiso decidido de las CCAA con la prevención cardiovascular; muestran una excesiva variabilidad en las políticas de prevención de la enfermedad cardiovascular entre las distintas CCAA.

En definitiva, es necesario continuar y reforzar las iniciativas actualmente en marcha, emprendidas por el CEIPC, para extender la implantación de una guía de prevención cardiovascular consensuada, evitando la confusión y la excesiva heterogeneidad en la práctica clínica. Asimismo, recomendamos a las autoridades sanitarias autonómicas impulsar el registro de los principales factores de riesgo en la HCE y la valoración del RCV, haciendo un énfasis especial en los estilos de vida. El Sistema Nacional de Salud tiene el reto de armonizar los indicadores de valoración y control del RCV y promover la inclusión de los mismos en los sistemas de incentivos de los profesionales de Atención Primaria. 


\section{repisälud}

\section{Agradecimientos}

A Carlos Martín (Antrópica) por su colaboración en la recogida, procesamiento y análisis de los datos. A Isabel San Andrés (Incimed) por su colaboración en la elaboración del primer borrador del manuscrito y en la búsqueda de bibliografía, confección de las tablas y maquetación de la versión final del documento. A todas las personas que hicieron posible la recogida de la información del estudio $\mathrm{y}$, en particular, a los referentes de prevención cardiovascular de las Comunidades Autónomas que cumplimentaron el cuestionario, entre los que se encuentran los siguientes: Gustavo Rodríguez Roca, Juan Fernández Martín, José Carlos Del Castillo Rodríguez, Sebastián Calero, Luis Lozano Mera, Enrique Martín Riobó, Carmen Lama Herrera y Salvador Tranche.

\section{Financiación}

Este estudio ha sido financiado por una beca no condicionada de MSD. El estudio fue coordinado por el CEIPC. Los autores tuvieron pleno acceso a todos los datos y fueron los responsables últimos de los contenidos del manuscrito y de la decisión de remitirlo para publicación.

\section{Conflictos de interés}

Miguel Ángel Royo Bordonada recibió honorarios como ponente de MSD en 2011. El resto de los autores no declaran conflictos de interés.

\section{Contribución de los autores}

Un comité de redacción, integrado por Miguel Ángel Royo Bordonada, José María Lobos, Fernando Villar, Carmen de Pablo, Pedro Armario y Ana de Santiago, se encargó de elaborar el borrador del manuscrito. Todos los autores participaron en la concepción y el diseño del estudio y en la discusión de los resultados. Todos los autores han leído y aprobado el documento final.

\section{Referencias Bibliográficas}




\section{repisälud}

1. Villar F, Banegas JR, de Mata Donado J, Rodríguez Artalejo F. Las enfermedades cardiovasculares y sus factores de riesgo en España: hechos y cifras. Informe SEA 2007. Sociedad Española de Arterioesclerosis. Disponible en: http://www.searteriosclerosis.org/resources/archivosbd/clinica_investigacion/4d34a5f3ab9cb226e076 bb3b11abf587.pdf

2. Propuesta de Estrategia en Cardiopatía Isquémica del Sistema Nacional de Salud. Secretaría General de Sanidad - Agencia de Calidad del Sistema Nacional de Salud. Madrid: Ministerio de Sanidad y Consumo; 2006 (Consultado en 2009). Disponible en: http://www.msps.es/organizacion/sns/planCalidadSNS/ec_pnc04.htm

3. Flores-Mateo G, Grau M, O'Flaherty M, Ramos R, Elosua R, Violan-Fors C, et al. Análisis de la disminución de la mortalidad por enfermedad coronaria en una población mediterránea: España 1988-2005. Rev Esp Cardiol. 2011;64:988-96.

4. Banegas JR, Graciani A, Guallar-Castillón P, León-Muñoz LM, Gutiérrez-Fisac JL, López-García E, et al. Estudio de Nutrición y Riesgo Cardiovascular en España (ENRICA). Madrid: Universidad Autónoma de Madrid; 2011.

5. Llisterri Caro JL, Rodríguez Roca GC, Alonso Moreno FJ, Prieto Díaz MA, Banegas Banegas JR, Gonzalez-Segura Alsina D; en representación del Grupo de Trabajo de Hipertensión Arterial de la Sociedad Española de Atención Primaria (Grupo HTA/SEMERGEN) y de los investigadores del Estudio PRESCAP 2010. Control de la presión arterial en población hipertensa española atendida en Atención Primaria. Estudio PRESCAP 2010. Med Clin (Barc). 2012 Mar 19. [Epub ahead of print]

6. Abellán Alemán J, Ruilope Urioste LM, Leal Hernández M, Armario García P, Tiberio López G, Martell Claros N. Control de los factores de riesgo cardiovascular en pacientes con ictus atendidos en Atención Primaria en España. Estudio ICTUSCARE. Med Clin (Barc). 2011 Mar 26;136(8):329-35. 


\section{repisälud}

7. Gutiérrez-Fisac JL, Guallar-Castillón P, León-Muñoz LM, Graciani A, Banegas JR, RodríguezArtalejo F. Prevalence of general and abdominal obesity in the adult population of Spain, 2008-2010: the ENRICA study. Obes Rev. 2012;13:388-92.

8. Soriguer F, Goday A, Bosch-Comas A, Bordiú E, Calle-Pascual A, Carmena R, et al. Prevalence of diabetes mellitus and impaired glucose regulation in Spain: the Di@bet.es Study. Diabetologia. 2012;55:88-93.

9. Grau M, Elosua R, Cabrera de León A, Guembe MJ, Baena-Díez JM, Vega Alonso T, et al. Factores de riesgo cardiovascular en España en la primera década del siglo xxi: análisis agrupado con datos individuales de 11 estudios de base poblacional, estudio DARIOS. Rev Esp Cardiol. 2011;64:295-304.

10. Baena-Díez JM, Félix FJ, Grau M, Cabrera de León A, Sanz H, Leal M, et al. Tratamiento y control de los factores de riesgo según el riesgo coronario en la población española del estudio DARIOS. Rev Esp Cardiol. 2011;64:766-73.

11. Cooney MT, Cooney HC, Dudina A, Graham IM. Assessment of cardiovascular risk. Curr Hypertens Rep. 2010;12:384-93.

12. de la Peña Fernández A, Roca Villanueva B, Cuende Melero I, Calabuig Alborch JR, Montes Santiago J, Muñoz Rodríguez M, et al, en representación del grupo CIFARC. Efecto de una intervención global sobre el control integral de múltiples factores de riesgo en pacientes con alto o muy alto riesgo cardiovascular. Estudio CIFARC 2. Rev Clin Esp. 2007;207:112-20.

13. Ministerio de Sanidad y Consumo. Campaña 2007- Prevención de enfermedades cardio y cerebrovasculares. Disponible en: http://www.msc.es/campannas/campanas07/cardiovascular3.htm 


\section{repisälud}

14. Maiques Galán A, Brotons Cuixart C, Villar Álvarez F, Lobos-Bejarano JM, Torcal Laguna J, Orozco-Beltrán D, et al. Recomendaciones preventivas cardiovasculares PAPPS del 2009. PAPPS; Actualización 2009.

15. Comité Español Interdisciplinario para la Prevención Cardiovascular (CEIPC). (Consultado en Abril 2012). Disponible en: www.ceipc.org

16. UNICEF. VIPP: Visualisation in Participatory Planning. Dhaka, Bangladesh: UNICEF; 1993.

17. McGee H, Morgan K \& Burke H, Royal College of Surgeons in Ireland. Implementation of the 4th Joint Societies Task Force Guidelines on Cardiovascular Disease Prevention in Clinical Practice. Evaluating implementation across 13 European countries. Report for the Prevention Implementation Committee, European Association of Cardiovascular Prevention and Rehabilitation. Royal College of Surgeons in Ireland; 2011.

18. Catalá-López, F, Sanfélix-Gimeno G, García-Torres C, Ridao M, Peiró S. Control of arterial hypertension in Spain: a systematic review and meta-analysis of 76 epidemiological studies on 341.632 participants. J Hypertens. 2012;30:168-176.

19. Redon J, Brunner HR, Ferri C, Hilgers KJ, Kolloch R, Van Montfranschs G. Practical solutions to the challenges of uncontrolled hypertension: a white paper. J Hypertens 2008;26 (Suppl 4):S1-S14.

20. Banegas JR, Jovell A, Abarca B, Aguilar Diosdado M, Aguilera L, Aranda P, et al. Hipertensión arterial y política de salud en España. Med Clin (Barc). 2009;132:222-229.

21. Lobos JM Royo-Bordonada MA, Brotons C, Alvarez-Sala L, Armario P, Maiques A, et al. Guía Europea de Prevención Cardiovascular en la Práctica Clínica. Adaptación Española del CEIPC 2008. Rev Esp Salud Publica. 2008;82:581-616. 


\section{repisälud}

22. Encuesta Nacional de Salud 2006. Consumo de tabaco en población de 16 años y más. INE; 2008.

Disponible

en:

http://www.ine.es/jaxi/menu.do?type=pcaxis \&path=/t15/p419/a2006/p07/\&file=pcaxis

23. Bertomeu V, Cordero A, Quiles J, Mazón P, Aznar J, Bueno H. Control de los factores de riesgo y tratamiento de los pacientes con cardiopatía isquémica: registro TRECE. Rev Esp Cardiol. 2009;62:807-11.

24. Kotseva K, Wood D, De Backer G, De Bacquer D, Pyörälä K, Keil U, et al. EUROASPIRE III: a survey on the lifestyle, risk factors and use of cardioprotective drug therapies in coronary patients from 22 European countries. Eur J Cardiovasc Prev Rehabil. 2009; 16: 121-137.

25. Estrategia Euskadi Libre del Humo del Tabaco. Osakidetza; 2010. Disponible en: http://www.osakidetza.euskadi.net/r85-

ckserv01/es/contenidos/nota_prensa/prensasanidad198/es_ps/adjuntos/euskadisinhumo.pdf

26. Redondo A, Subirana I, Ramos R, Solanas P, Sala J, Masiá R, et al. Tendencias en la práctica de actividad física en el tiempo libre en el periodo 1995-2005 en Girona. Rev Esp Cardiol. 2011;64:9971004.

27. Palacios-Ceña D, Alonso-Blanco C, Jiménez-Garcia R, Hernández-Barrera V, Carrasco-Garrido P, Pileño-Martinez E, et al. Time trends in leisure time physical activity and physical fitness in elderly people: 20 year follow-up of the Spanish population national health survey (1987-2006). BMC Public Health. 2011;11:799.

28. Meseguer CM, Galán I, Herruzo R, Rodríguez-Artalejo F. Tendencias de actividad física en tiempo libre y en el trabajo en la Comunidad de Madrid, 1995-2008. Rev Esp Cardiol. 2011;64:21-7. 


\section{repisälud}

29. Salcedo V, Gutiérrez-Fisac JL, Guallar-Castillón P, Rodríguez-Artalejo F. Trends in overweight and misperceived overweight in Spain from 1987 to 2007. Int J Obes (Lond). 2010;34:1759-65.

30. Bouillon K, Singh-Manoux A, Jokela M, et al. Decline in low-density lipoprotein cholesterol concentration: lipid-lowering drugs, diet, or physical activity? Evidence from the Whitehall II study. Heart. 2011;97:923-30. 